\title{
Comparative gene expression profiling of muscle reveals potential candidate genes affecting drip loss in pork
}

\author{
Xueyan Zhao ${ }^{\dagger}$, Cheng Wang ${ }^{\dagger}$, Yanping Wang, Haichao Lin, Huaizhong Wang, Hongmei Hu and Jiying Wang* ${ }^{*}$
}

\begin{abstract}
Background: Drip loss is a key aspect of meat quality. Transcriptome profiles of muscle with divergent drip loss would offer important insight into the genetic factors responsible for the trait. In this study, drip loss and other meat quality traits of 28 purebred Duroc pigs were measured, muscles of these individuals were RNA sequenced, and eight individuals with extremely low and high drip loss were selected for analyzing their transcriptome differences and identifying potential candidate genes affecting drip loss.

Results: As a result, 363 differentially expressed (DE) genes were detected in the comparative gene expression analysis, of which 239 were up-regulated and 124 were down-regulated in the low drip loss group. The DE genes were further filtered by correlation analysis between their expression and drip loss values in the 28 Duroc pigs measured and comparison of them with QTLs affecting drip loss. Consequently, of the 363 DE genes, 100 were identified as critical DE genes for drip loss. Functional analysis of these critical DE genes revealed some GO terms (extracellular matrix, cell adhesion mediated by integrin, heterotypic cell-cell adhesion), pathway (ECM-receptor interaction), and new potential candidate genes (TNC, ITGA5, ITGA11, THBS3 and CD44) which played an important role in regulating the variation of drip loss, and deserved to carry further studies to unravel their specific mechanism on drip loss.

Conclusions: Our study revealed some GO terms, pathways and potential candidate genes affecting drip loss. It provides crucial information to understand the molecular mechanism of drip loss, and would be of help for improving meat quality of pigs.
\end{abstract}

Keywords: Pig, Meat quality, RNA sequencing, Differentially expressed genes

\section{Background}

Meat quality can be evaluated by multiple indicators, such as water holding capacity (WHC), meat color, intramuscular fat (IMF) and tenderness. Among these, WHC, i.e. the ability of meat to retain water, is arguably one of the most important commercially interesting indicators, which directly influences the appearance, sensory quality, and the value of meat produced. WHC is often predicted by drip loss, which is defined as the fresh meat loss rate under gravity at $4{ }^{\circ} \mathrm{C}$ for $24 \mathrm{~h}$ [1]. Moreover, WHC is closely

\footnotetext{
* Correspondence: jnwangjiying@163.com

${ }^{\dagger}$ Xueyan Zhao and Cheng Wang contributed equally to this work.

Shandong Provincial Key Laboratory of Animal Disease Control and Breeding, Institute of Animal Science and Veterinary Medicine, Shandong Academy of Agricultural Sciences, Jinan 250100, China
}

related to other meat quality indicators, including meat color, $\mathrm{pH}$, and IMF $[2,3]$.

Large phenotypic differentiation of drip loss in musculus longissimus dorsi (LD) was observed in the previous studies [4, 5]. Apart from environmental factors, this wide range could be explained by genetic factors which directly affect the muscle characteristics.Therefore, it is considered as a target trait in some breeding schemes. However, drip loss exhibits low to moderate heritability, varying from 0.08 to 0.30 depending on the measurement methods or breeds [6-8], and it is difficult to improve such traits using conventional breeding methods. Therefore, the development of valuable molecular markers or the identification of key genes related to drip loss would provide new opportunities for decreasing drip loss of pork using molecular breeding technologies.

(c) The Author(s). 2019 Open Access This article is distributed under the terms of the Creative Commons Attribution 4.0 International License (http://creativecommons.org/licenses/by/4.0/), which permits unrestricted use, distribution, and reproduction in any medium, provided you give appropriate credit to the original author(s) and the source, provide a link to the Creative Commons license, and indicate if changes were made. The Creative Commons Public Domain Dedication waiver (http://creativecommons.org/publicdomain/zero/1.0/) applies to the data made available in this article, unless otherwise stated. 
Drip loss, like the other meat quality traits, is a complex quantitative trait and influenced by many genes. Until now, a variety of genetic approaches have been applied to elucidate the genetics that underlies this trait, such as identifying quantitative trait loci (QTLs), candidate genes and other markers. To date, 1115 QTLs related to WHC and drip loss (http://www.animalgenome. org/cgi-bin/QTLdb/SS/index, Release 39, Aug 22, 2019) were reported in different pig populations. However, these QTLs were identified mostly via genome scan based on microsatellite genotyping, and generally large genomic regions. Further fine-mapping studies to find causal genes are challenging. Recently, with the development of highdensity SNP chips and genome sequencing, genome-wide association studies (GWAS) have been used as a more precise method for identifying the genomic regions and markers associated with drip loss [9-11]. Moreover, in the studies of candidate genes, two major genes, RYR1 [12] and PRKAG3 [13], have been demonstrated to control drip loss for pale, soft and exudative (PSE) and dark, firm and dry (DFD) meat, respectively, and one causal gene, PHKG1 [14], for normal quality meat. Despite these progress, the knowledge of the genetic factors underlying drip loss remains very incomplete.

Gene expression patterns may explain a high degree of the observed phenotypic differences in a given tissue. Transcriptomics, based on RNA sequencing (RNA-seq), enables high throughput screening of expressed genes, and is a powerful tool for exploring the genetic architecture of complex traits. Previous studies examining the transcriptome of the porcine LD muscle have successfully identified differentially expressed (DE) genes affecting backfat thicknesses [15], IMF [16] and drip loss [17, 18]. Although some DE genes have been identified, the results are not consistent among studies. In this study, the LD muscles of individuals with extremely low and high drip loss were sampled from a purebred Duroc pig population, and RNA-seq technology was applied to screen DE genes, followed by functional analysis to identify plausible candidate genes affecting drip loss. This study provides valuable information for understanding the molecular basis underlying drip loss of pigs.

\section{Results}

\section{Drip loss variation among Duroc pigs}

Twenty-eight Duroc pigs were used in this study. Their detailed information of carcass and meat quality traits is presented in Table 1. It can be seen that these pigs had good carcass quality, and their lean meat rate, backfat thickness and large eye muscle area were $66.90 \%, 16.30$ $\mathrm{mm}$ and $46.94 \mathrm{~cm}^{2}$ in average, respectively. And these pigs also showed to have normal meat quality according to the traits of $\mathrm{pH}_{1}, \mathrm{pH}_{24}$ as well as $\mathrm{L}^{*}, \mathrm{a}^{*}$ and $\mathrm{b}^{*}$, whose averages were $6.49,5.72,42.78,5.72$, and 10.63 , respectively.

Specifically for the values of target trait, drip loss, they were normally distributed with $P$ value of 0.1787 for Shapiro-Wilk normality test, and the average was $2.36 \%$, with the minimum and maximum of 1.63 and $3.67 \%$, respectively. Four individuals with extremely low drip loss ( 2 male and 2 female) and four with extremely high drip loss (2 male and 2 female) were selected for further genome-wide gene expression analysis. No sib or halfsib relationship existed among the 8 individuals selected for the transcriptome analysis.

The detailed phenotypic information of the eight individuals is provided in Additional file 1: Table S1 and Table 1. And the distribution of drip loss among the 28 individuals is demonstrated in Fig. 1. Compared between the two groups, the average of the high drip loss group (3.1\%) was significantly greater than that of the low drip loss group $(1.69 \%)$ with $P$ value of 0.002 . It is important to note that besides drip loss, shear force, and $\mathrm{a}^{*}$ also

Table 1 Phenotypic information of drip loss and other carcass and meat quality traits

\begin{tabular}{|c|c|c|c|c|}
\hline Traits & Total 28 samples measured & Four samples of Low drip loss group & Four samples of High drip loss group & $P$ value \\
\hline Drip loss (\%) & $2.36 \pm 0.53$ & $1.69 \pm 0.06$ & $3.03 \pm 0.09$ & 0.002 \\
\hline Weight (kg) & $108.30 \pm 6.00$ & $107.3 \pm 1.68$ & $107.5 \pm 2.83$ & 0.285 \\
\hline Backfat (mm) & $16.30 \pm 4.49$ & $14.6 \pm 3.68$ & $15.1 \pm 2.12$ & 0.198 \\
\hline Eye muscle area $\left(\mathrm{cm}^{2}\right)$ & $46.94 \pm 5.38$ & $53.8 \pm 3.84$ & $50.2 \pm 1.84$ & 0.107 \\
\hline Percentage of lean meat (\%) & $66.90 \pm 2.57$ & $68.9 \pm 2.47$ & $69.1 \pm 2.05$ & 0.366 \\
\hline $\mathrm{pH}_{1}$ & $6.49 \pm 0.22$ & $6.39 \pm 0.09$ & $6.79 \pm 0.01$ & 0.053 \\
\hline $\mathrm{pH}_{24}$ & $5.72 \pm 0.14$ & $5.79 \pm 0.11$ & $5.67 \pm 0.02$ & 0.078 \\
\hline IMF (\%) & $2.36 \pm 0.71$ & $2.05 \pm 0.48$ & $2.45 \pm 0.17$ & 0.264 \\
\hline Shear force(N) & $45.80 \pm 10.96$ & $59.8 \pm 10.26$ & $44.85 \pm 7.85$ & 0.025 \\
\hline$L^{*}$ & $42.78 \pm 1.82$ & $43.09 \pm 1.14$ & $43.93 \pm 0.74$ & 0.590 \\
\hline$a^{*}$ & $5.72 \pm 0.78$ & $4.94 \pm 0.34$ & $5.94 \pm 0.30$ & 0.027 \\
\hline$b^{*}$ & $10.63 \pm 0.80$ & $10.27 \pm 0.41$ & $11.16 \pm 0.04$ & 0.407 \\
\hline
\end{tabular}

The results are showed by mean \pm standard deviation 


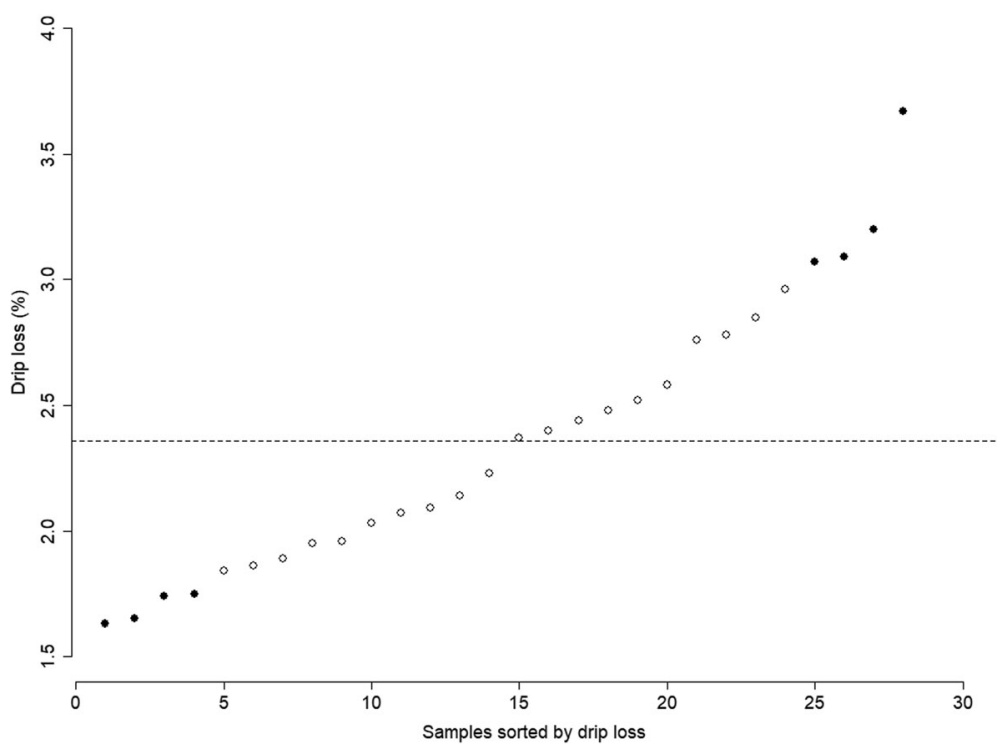

Fig. 1 Distribution of the low and high drip loss samples selected for RNA-seq in the 28 Duroc pigs. The solid dots indicate the samples selected for RNA-seq

show significant differences between the two groups, demonstrating that there are close correlations between drip loss and these traits.

\section{Summary statistics for RNA-seq data}

Using paired-end RNA-seq approach, we sequenced the transcriptome of LD muscle of the 28 Duroc pigs. In total, we generated 52.43-86.93 million paired-end raw reads per sample. Prior to assembly, low quality reads, which was $2.31 \%$ of the raw reads, were removed. As a result, an average of 62.56 million cleaned reads were produced for the 28 samples after filtering. A summary of the sequencing results is shown in Additional file 2: Table S2. After alignment, an average of 90.36 and $4.01 \%$ of the cleaned reads could be mapped to the recent Sus scrofa genome (Sscrofa 11.1) with unique position and multiple positions respectively. Additionally, out of the reads mapped to the reference genome, 91.58\% fell in annotated exons, 3.86\% were located in introns, and the remaining $4.56 \%$ were assigned to intergenic regions. Unmapped or multiposition matched reads were excluded from further analyses.

\section{Expression profiling of muscle with low and high drip loss}

A total of 26,918 genes were detected for samples sequenced through alignment with the recent Sus scrofa genome. After filtering those genes with a minimal number of reads $(\leq 1)$ in more than one third of the 28 individuals, there were 18,064 genes expressed, whose normalized expression values by DESeq 2 is presented in Additional file 3: Table S3. And further analyses were conducted based on them. Out of the 18,064 genes, 17, 071 were included in Ensembl pig genome database, while the other 993 were unannotated in Sus scrofa reference genome (Sscrofa 11.1) and potential novel genes (Additional file 4: Table S4).

Using DESeq2, the differential expression analysis between low and high drip loss groups was performed. According to the empirical studies, FDR adjustment of $P$ value $<0.05$ and fold change $\geq 2$ or fold change $<0.5$, were used to reduce false positive rate of DE genes. As a result, a total of 363 genes showed significant expression differences between the two groups, and 239 and 124 genes were up-regulated and down-regulated in the low drip loss group, respectively. Their detailed information is provided in Additional file 5: Table S5. Figure 2 shows the heatmap of these DE genes, from which, it can be seen that the expression patterns are consistent within groups and different between groups.

\section{Correlation between expression of DE genes and their drip loss values}

To filter the DE genes detected, Pearson correlation analysis was conducted between expressions of these DE genes and their drip loss values in the data set of 28 Duroc pigs measured in this study. As shown in Fig. 3, expressions of all the up-regulated DE genes were negatively correlated with their drip loss (correlation coefficients ranging from 0.00 up to 0.69 ), while those of all but two of the down-regulated DE genes were positively correlated (correlation coefficients ranging from -0.65 up to - 0.09). Furthermore, significance test of correlation analysis indicated that there were 209 genes, 


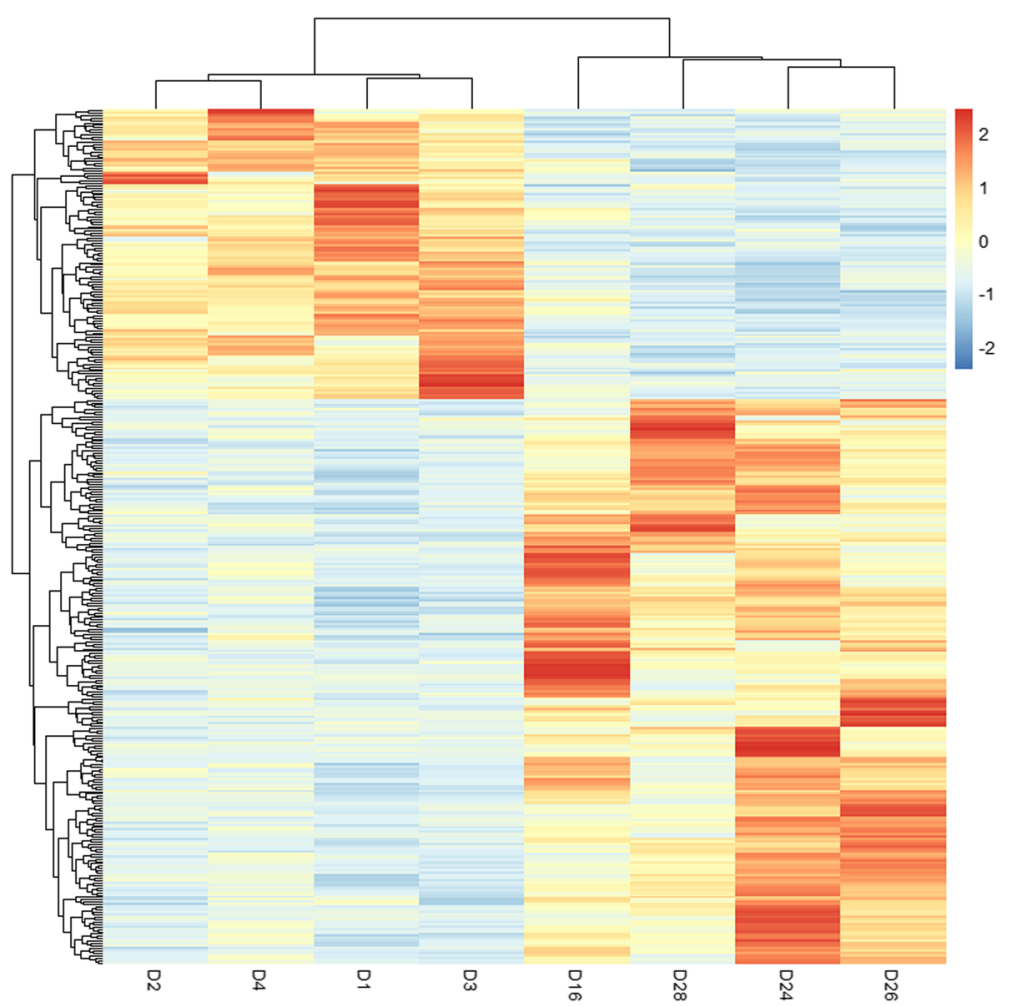

Fig. 2 Heatmap for DE genes. Columns and rows indicate pigs and DE genes, respectively. Color scale represents $\log _{10}$ (normalized expression values of DE genes). Red and blue represent up- and down-regulated DE genes, respectively. DE genes, differentially expressed genes

including 122 down-regulated and 87 up-regulated genes, whose expressions and the drip loss values were significantly correlated $(p<0.05)$. The detailed information of these significantly correlated DE genes is presented in Additional file 6: Table S6.

\section{Comparison of DE genes with QTLs affecting drip loss}

We further filtered the DE genes detected by comparing the DE genes with the QTLs affecting drip loss collected in the pig QTL database (http://www.animalgenome.org/ cgi-bin/QTLdb/SS/index, Release 39, Aug 22, 2019). In

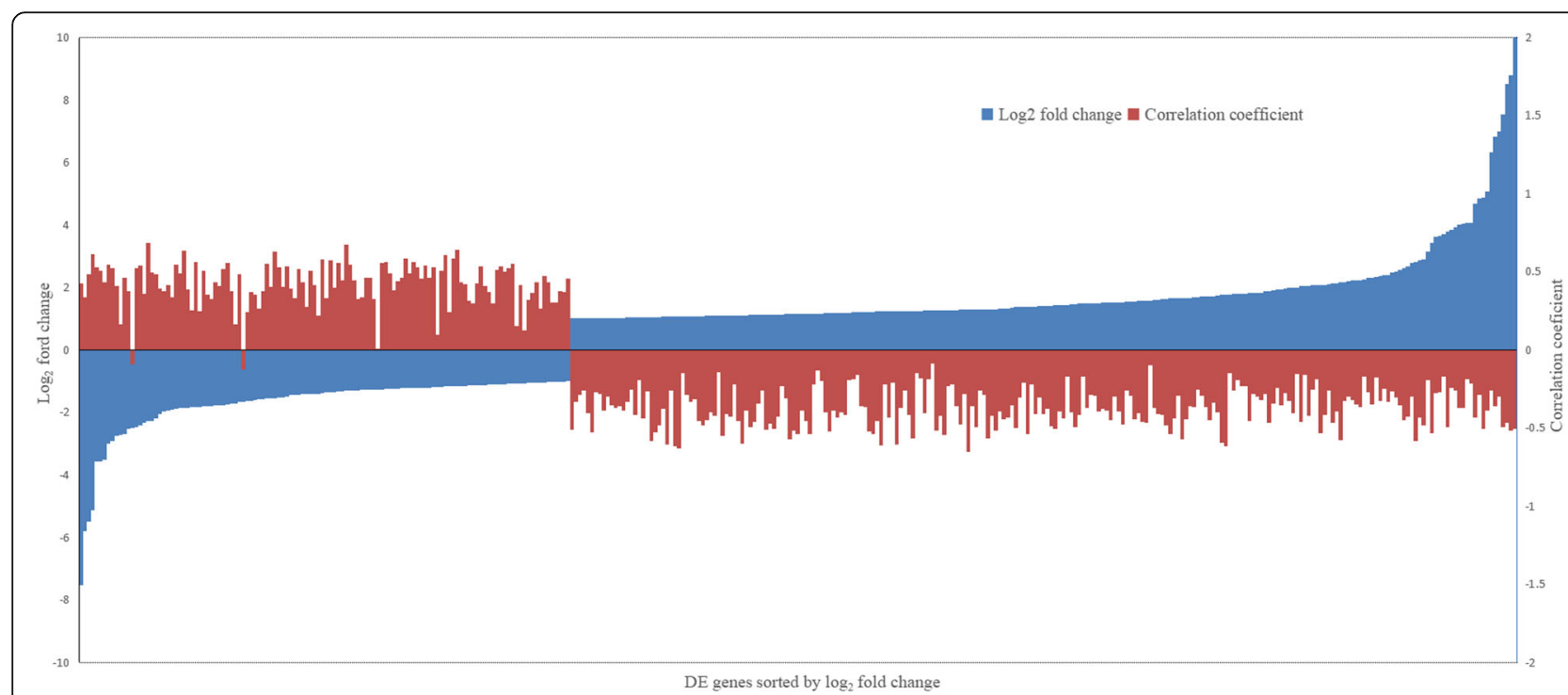

Fig. 3 Pearson's correlation coefficients between expressions of DE genes detected and their drip loss values in the 28 Duroc pigs measured in this study. DE genes, differentially expressed genes 
total, 1115 QTLs in the pig QTL database were reported to relate with drip loss or WHC. Since some QTLs have identical physical positions or no exact physical position, 310 QTLs with unique physical position were used to compare with DE genes. As a result, $192 \mathrm{DE}$ genes were mapped to the 60 of these 310 QTLs (Additional file 7: Table S7). Further enrichment analysis by Fisher's exact test indicated that $\mathrm{DE}$ genes were significant enriched in QTLs affecting drip loss $(P$ value $<2.2 \mathrm{e}-16)$. High proportion of DE genes mapping in QTLs affecting the trait proofed that the DE genes detected in the study were more likely to be candidate genes affecting drip loss.

\section{Functional analysis of critical DE genes detected for drip loss}

Combining the above two filtering conditions, of the 363 DE genes, we identified 100 critical DE genes for drip loss (Additional file 8: Table S8). They located in the QTLs related to drip loss, and their expressions were significantly correlated with drip loss values in 28 Duroc pigs. To have a functional view of them, we carried out Gene Ontology (GO) and Kyoto Encyclopedia of Genes and Genomes (KEGG) enrichment analysis for them, and determined 12 significantly enriched GO terms (Additional file 9: Table S9) and three pathways (Additional file 10: Table S10) with $P$ value $<0.01$. Among the 12 enriched GO terms, 10 terms were belonged to biological process
(BP) category and involved in cell adhesion, wound healing and amino acid transport, meanwhile the other ones, cell surface and extracellular matrix, were belonged to cellular component (CC) category.

Figure 4 illustrates part of critical DE genes involved in the nine significantly enriched GO terms and three pathways which may related to drip loss. Among these genes, TNC ( $q$ value $=2.90 \mathrm{E}-03, \mathrm{FC}=2.98)$, ITGA5 $(q$ value $=4.96 \mathrm{E}-03, \mathrm{FC}=2.35)$, ITGA11 $(q$ value $=7.88 \mathrm{E}-04$, $\mathrm{FC}=2.17), C D 44(q$ value $=2.77 \mathrm{E}-04, \mathrm{FC}=2.35)$, EFNA1 $(q$ value $=2.61 \mathrm{E}-02, \mathrm{FC}=2.14), \operatorname{CSPG4}(q$ value $=4.05 \mathrm{E}-$ $07, \mathrm{FC}=2.45)$, and THBS3 $(q$ value $=2.27 \mathrm{E}-07, \mathrm{FC}=$ 2.35) were involved in more than three GO terms or pathways, implying there could be possible interactions among these genes. We further constructed proteinprotein interaction (PPI) network analysis using Search Tool for the Retrieval of Interacting Genes (STRING). Consequently, a PPI network, consisting of five core proteins TNC, ITGA5, ITGA11, THBS3 and CD44, was constructed, suggesting the five genes biologically connected (Fig. 3). Table 2 presents the detailed information of the five candidate genes.

\section{Discussion}

Understanding the molecular mechanisms underlying drip loss is a essential way to improve the economic value of pork. In this study, to identify candidate genes

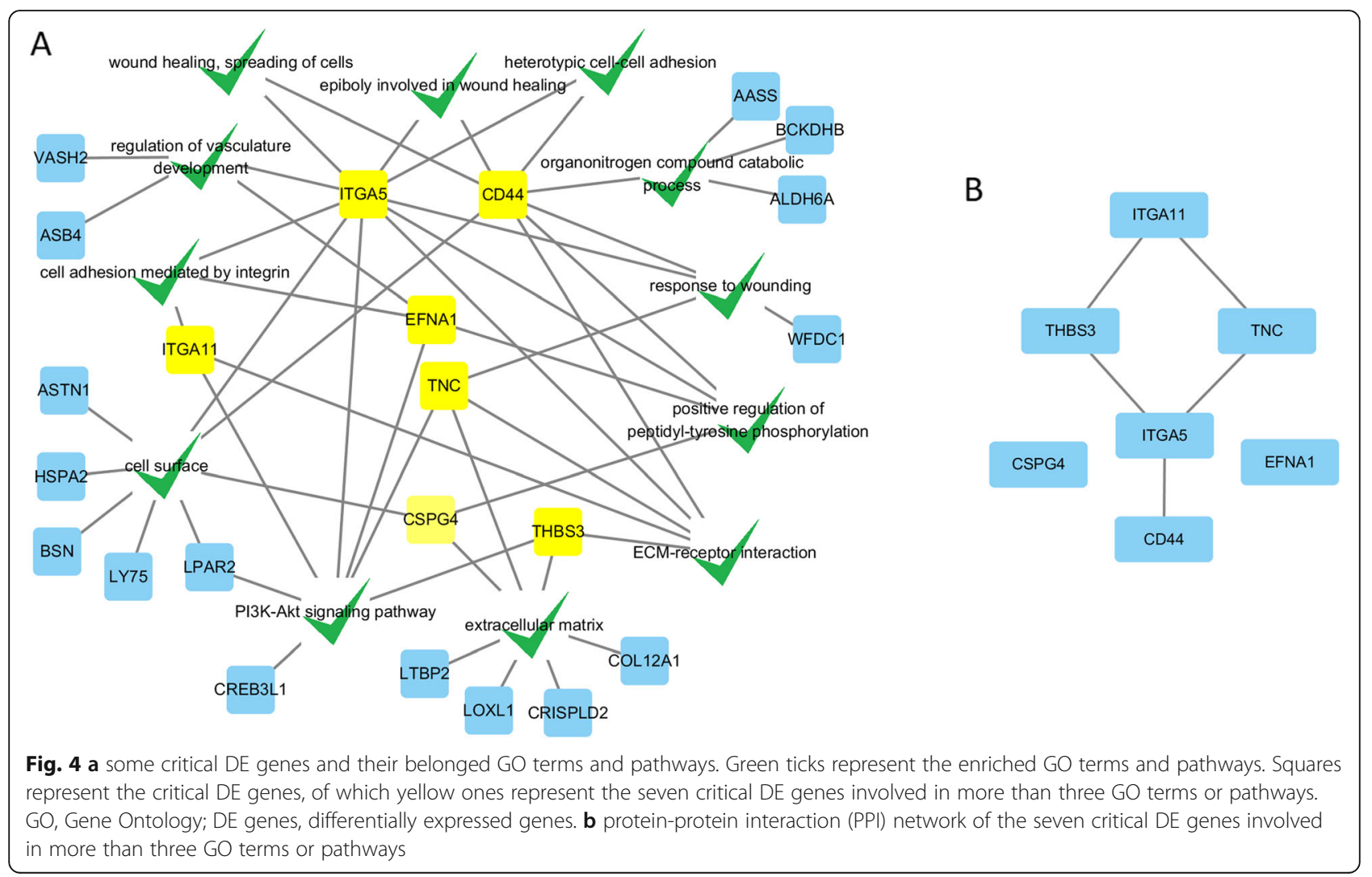


Table 2 List of some potential candidate genes between low and high drip loss groups

\begin{tabular}{|c|c|c|c|c|c|c|c|}
\hline \multicolumn{2}{|l|}{ Gene information } & \multicolumn{2}{|c|}{$\begin{array}{l}\text { Differential expression values } \\
\text { between two groups }\end{array}$} & \multicolumn{2}{|l|}{$\begin{array}{l}\text { Pearson correlation in } 28 \\
\text { Duroc pigs }\end{array}$} & \multirow[t]{2}{*}{ QTL_ID } & \multirow[t]{2}{*}{ Gene description } \\
\hline Ensembl_ID & Gene name & $\begin{array}{l}\log _{2} \text { fold } \\
\text { change }\end{array}$ & $\begin{array}{l}\text { FDR adjusted } \\
P \text { value }\end{array}$ & Correlation coefficient & $P$ value & & \\
\hline ENSSSCG00000000293 & ITGA5 & 2.19 & $7.23 \mathrm{E}-05$ & -0.4754 & $1.06 \mathrm{E}-02$ & 3805,9847 & Integrin subunit alpha 5 \\
\hline ENSSSCG00000004961 & ITGA11 & 2.17 & $5.20 \mathrm{E}-06$ & -0.4936 & 7.60E-03 & 7936,7784 & Integrin subunit alpha 1 \\
\hline ENSSSCG00000013297 & CD44 & 2.35 & $1.20 \mathrm{E}-06$ & -0.4341 & $2.10 \mathrm{E}-02$ & $\begin{array}{l}86,2937,3780 \\
21,848,38,075\end{array}$ & CD44 molecule \\
\hline ENSSSCG00000005494 & TNC & 2.98 & $3.32 \mathrm{E}-05$ & -0.4659 & $1.24 \mathrm{E}-02$ & 7936,8419 & Tenascin C \\
\hline ENSSSCG00000006524 & THBS3 & 2.35 & 2.27E-07 & -0.6102 & $5.65 \mathrm{E}-04$ & $3847,38,084$ & Thrombospondin 3 \\
\hline
\end{tabular}

influencing drip loss of pigs with normal meat quality, we selected extremely low and high drip loss samples from a high number of Duroc pigs with the same environment, performed a comparative expression profiling analysis, and detected $363 \mathrm{DE}$ genes (Additional file 5: Table S5) between the two groups with extremely low and high drip loss. The DE genes were further filtered by correlation analysis between their expression and drip loss value in the 28 Duroc pigs measured and comparison of them with QTLs affecting drip loss to reduce false positive. Consequently, we identified 100 critical DE genes affecting drip loss (Additional file 8: Table S8), which located in the QTLs related to drip loss, and whose expressions were significantly correlated with drip loss values in 28 Duroc pigs. These critical DE genes would provide crucial information to understand the molecular mechanisms underlying drip loss.

Some enriched GO terms and pathways related to extracellular matrix, such as extracellular matrix (GO: 0031012, extracellular matrix) and ECM-receptor interaction (KEGG:ssc04512, ECM-receptor interaction), were identified in the functional analysis of the critical DE genes detected. Extracellular matrix consists of many proteins, which interact with each other and form a super molecular network to withstand and transmit the contractile forces generated by muscle fibers [19], and thus may play important role in influencing drip loss. Besides extracellular matrix, integrins have been proposed to attach the cytoskeleton to the extracellular matrix, and have impact on the formation of drip channels in pork [20]. Previous works have indicated that there was a reverse correlation between postmortem degradation of integrins and drip loss [21, 22]. In our result, two enriched GO terms related to integrins and adhesion, cell adhesion mediated by integrin (GO:0033627) and heterotypic cell-cell adhesion (GO:0034113), were also discovered. Therefore, extracellular matrix, cell adhesion mediated by integrin and heterotypic cell-cell adhesion are potential candidate GO terms, while ECMreceptor interaction are potential candidate pathway affecting drip loss.
Among the critical DE genes detected in the study, TNC, ITGA5, ITGA11, THBS3 and CD44 were not only repeatedly discovered as the important genes in the enriched GO terms and pathways (Fig. 4), but coded the five core proteins in the PPI network analysis. TNC encodes an extracellular matrix glycoprotein, interacts with several other extracellular matrix molecules and cellsurface receptors, thus affecting tissue architecture, tissue resilience and cell responses. It is significantly upregulated in response to tissue injury [23]. The product of ITGA5 and ITGA11 belong to the integrin alpha chain family. Integrins are heterodimeric integral membrane proteins composed of an alpha subunit and a beta subunit that function in cell surface adhesion and signaling [24]. THBS3 is an adhesive glycoprotein that is involved in cell-cell and cell-matrix interactions. Newly study by Schips [25] demonstrated that THBS3 promotes sarcolemmal destabilization by reducing integrin function, augmenting disease-induced decompensation. CD44 is a multifunctional transmembrane glycoprotein that serves as a cell-surface receptor for a number of ECM proteins [26]. It can be seen that the main function of the five genes are related to extracellular matrix and integrins, which as mentioned above, have impact on the formation of drip loss. All the above proofs that the five genes are potential candidate genes affecting drip loss.

Except potential candidate genes listed above, we also confirmed several known candidate genes influencing drip loss reported in the previous studies. Heat shock proteins (HSPs) HSPs belong to stress related proteins, and have been reported as potential biomarkers for several meat quality traits (WHC, tenderness, and colour) [27, 28]. In this study, several HSP genes, like HSPB1, HSPH1, HSPB7 and HSPA1L were found to not only differently express between the low and high drip loss groups, but significantly correlated in the correlation analysis in the 28 Duroc pigs. An improved abundance of these proteins may enable cells to overcome stressful conditions and leads to less fluid exuding from the cells. In addition, genes of solute carrier family (SLC), such as SLC37A4 and SLC3A2, have been reported to be 
promising candidate genes influencing drip loss trait $[18,29]$. In our study, SLC37A4 was differently expressed between divergent drip loss groups, significantly correlated in the large samples, and located in the QTL (QTL_ID 3806) related to drip loss. Hence, our study confirmed it to be a promising candidate gene for drip loss. Additionally, we identified several other genes of solute carrier family members, suggesting the importance of gene of solute carrier family in regulating drip loss.

\section{Conclusion}

In this study, transcriptome analysis based on RNA-seq technology was performed to evaluate the gene expression differences of muscle with divergent drip loss. A total of 363 DE genes were screened by DESeq 2 with 239 and 124 genes up-regulated and down-regulated in the low drip loss group, respectively. Combining the filtering conditions of correlation analysis and comparison with QTLs related to drip loss, we identified 100 critical DE genes affecting drip loss from the $363 \mathrm{DE}$ genes detected. Further functional analysis of the critical DE genes revealed some GO terms (extracellular matrix, cell adhesion mediated by integrin, heterotypic cell-cell adhesion), pathway (ECMreceptor interaction), and new potential candidate genes (TNC, ITGA5, ITGA11, THBS3 and CD44) which have impact on the variation of drip loss.

\section{Methods}

\section{Animals and phenotypic traits}

Thirteen male and 15 female purebred Duroc pigs from Jianghai Pig Breeding Co., Ltd. were used in our study. The pigs came from different sires and dams, and thus no sib and half-sib relationships were existed among them. The animals were reared indoors in a standard pig breeding farm and fed with diets formulated according to age under a standardized feeding regimen with free access to water. All pigs were slaughtered at an average weight of $108.29 \pm 6.00 \mathrm{~kg}$ (mean \pm standard deviation) in one batch in compliance with national guidelines. The pigs were stunned by electric shock, and the carcasses were exsanguinated, scalded and split at unconsciousness. Moreover, all pigs were free of the Halothane gene mutation (RYR1; C1843T), ensured by DNA testing prior to the experiment.

After slaughter, the backfat thickness (average thickness of the thickest of shoulder, the last rib and the lumbosacral junction) and eye muscle area (at the last rib) were measured using a vernier caliper. After splitting the left carcass into lean meat, fat, skin and bone, the percentage of lean meat was estimated by calculating the lean meat percentage of the sum of lean meat, fat, skin and bone. The meat quality traits were evaluated using samples taken from LD muscle of the left carcass.
Specifically, muscle $\mathrm{pH}$ at 45 min postmortem $\left(\mathrm{pH}_{1}\right)$ and $24 \mathrm{~h}$ postmortem $\left(\mathrm{pH}_{2}\right)$ were measured at the last 1 st and 2nd thoracic vertebrae using a portable $\mathrm{pH}$ meter (MATTHAUS PH-STAR, German) for meat. Drip loss was measured in triplicate per sample using three muscle samples about $10 \mathrm{~g}$ taken from the last 3rd and 4th thoracic vertebra within $60 \mathrm{~min}$ postmortem. Each one was weighed and placed into a plastic measuring device like a funnel with lid. After storage for $24 \mathrm{~h}$ at $4{ }^{\circ} \mathrm{C}$, the samples were dabbed with filter paper and reweighed. Drip loss was calculated as the percentage of weight loss based on the initial weight of the sample. Muscle sample was taken from the last 4th and 5th thoracic vertebrae, trimmed of external fat, minced, and dried for determination of IMF content using chloroform-methanol extraction method. IMF content was measured in triplicate per sample to ensure its accuracy. The CIE L* (lightness), $\mathrm{a}^{*}$ (redness), and $\mathrm{b}^{*}$ (yellowness) parameters were determined at the 1st lumbar vertebra within 60 min postmortem using a CR310 Minolta chromameter (Osaka, Japan). Shear force was also determined using samples taken from the 1st and 3rd lumbar vertebrae. Samples were firstly aged up to $4 \mathrm{~d}$ postmortem at $4{ }^{\circ} \mathrm{C}$, then heated in $80^{\circ} \mathrm{C}$ water-bath until the center temperature up to $70^{\circ} \mathrm{C}$. After cooled at room temperature, samples parallel to fiber axis were cut into 1 $\mathrm{cm}^{2}$ sections for measurement of shear force perpendicularly to muscle fibers using a digital muscle tenderness meter (C-LM3B, Northeast Agricultural University, Harbin, China). Shear force was the average of 15 measurements per sample.

Total RNA extraction, library construction and sequencing Immediately after slaughter, LD muscles were taken from the 4th thoracic vertebra from the last rib, put into tubes with RNAlater Stabilization Solution (Thermo Fisher, Waltham, MA, USA), and frozen at $-80^{\circ} \mathrm{C}$ for RNA extraction. Total RNA was isolated from the 28 samples using TRIzol reagent (Invitrogen, Life Technologies). The purity and concentration of total RNA were assessed by NanoDrop 1000 instrument (Thermo Fisher Scientific),) and Qubit ${ }^{\oplus}$ RNA Assay Kit in Qubit ${ }^{\bullet}$ 2.0 Flurometer (Life Technologies, CA, USA), and integrity was tested using the RNA Nano 6000 Assay Kit of the Bioanalyzer 2100 system (Agilent Technologies, CA, USA).

RNA libraries were constructed for the 28 pigs measured in the study. A total amount of $3 \mu \mathrm{g}$ RNA per sample was used as input material for the RNA-seq library preparations. Sequencing libraries were generated using NEBNext ${ }^{\oplus}$ Ultra $^{\mathrm{Tm}}$ RNA Library Prep Kit for Illumina ${ }^{\oplus}$ (NEB, USA) following the manufacturer's recommendations and index codes were added to attribute sequences to each sample. The library preparations were sequenced on an Illumina Hiseq platform and $150 \mathrm{bp}$ paired-end reads were generated. 


\section{Differential gene expression analysis}

To obtain clean reads, in-house perl scripts were used to discard adaptor sequences, reads containing ploy- $\mathrm{N}$ (the percentage of unknown bases is more than $10 \%$ in a read) and low quality reads from raw data (the percentage of low-quality bases of error rate $>1 \%$ is more than $50 \%$ in a read). Clean reads were then mapped to Sus scrofa reference genome (Sus scrofa 11.1, http://asia.ensembl.org/Sus_ scrofa/Info/Index) using HISAT2 [30] with default parameters. Then, featureCounts tool of subread package [31] was used to count read numbers mapped to each gene.

Differential expression analysis was performed using DESeq2 (v1.18.0) [32]. It provides statistical routines for determining differential expression in gene expression data using a model based on the negative binomial distribution. The resulting $P$ values were adjusted using the Benjamini and Hochberg's approach for controlling the false discovery rate (FDR). According to the empirical studies, two criteria, i.e., the adjusted $q$ value $<0.05$ and fold change (FC) $\geq 2$ or $F C \leq 0.5$, were used as cutoffs to obtain differentially expressed (DE) genes. Genes that meet the criteria were regarded as DE genes, and their heatmap was drawn by $\mathrm{R}$ package pheatmap (https:// cran.r-project.org/web/packages/pheatmap/).

\section{Filtering and functional characterization of DE genes}

Pearson correlation between the expression of DE genes and their drip loss values were analyzed using $\mathrm{R}$ package Hmisc (https://cran.r-project.org/web/packages/Hmisc/ index.html) in the data set of 28 Duroc pigs measured in this study. QTLs affecting WHC or drip loss trait were downloaded from the pig QTL database (http://www. animalgenome.org/cgi-bin/QTLdb/SS/index, Release 39, Aug 22, 2019), and compared with DE genes based on their physical position. DE genes filtered by the above two conditions were regarded as critical DE genes.

Gene Ontology (GO) and Kyoto Encyclopedia of Genes and Genomes (KEGG) pathway enrichment analyses of critical DE genes were conducted by $\mathrm{R}$ package "clusterProfiler" [33], which implemented hypergeometric model to select significant enriched GO term and KEGG pathway. GO terms and pathway with $P$ value less than 0.01 were considered to be significantly enriched.

\section{Supplementary information}

Supplementary information accompanies this paper at https://doi.org/10. 1186/s12863-019-0794-0.

Additional file 1: Table S1. Phenotypic information of carcass and meat quality traits for the low and high samples selected for transcriptome analysis.

Additional file 2: Table S2. Summary of sequencing reads and their alignments to Sus scrofa reference genome.

Additional file 3: Table S3. Expressed genes detected and their normalized expression values.
Additional file 4: Table S4. Potential novel genes detected in the study.

Additional file 5: Table S5. Detailed information of DE genes detected between low and high drip loss groups.

Additional file 6: Table S6. Correlation between expressions of DE genes and their drip loss values in the 28 Duroc pigs.

Additional file 7: Table S7. Comparison of DE genes and QTLS related to drip loss.

Additional file 8: Table S8. Detailed information of critical DE genes detected between low and high drip loss groups.

Additional file 9: Table S9. Significantly enriched GO terms of the critical DE genes detected between low and high drip loss groups.

Additional file 10: Table S10. Significantly enriched pathways of the critical DE genes detected between low and high drip loss groups.

\section{Abbreviations}

BP: Biological process; CC: Cellular component; DE: Differentially expressed; DFD: Dark, firm and dry; EM: Extracellular matrix; GO: Gene Ontology; GWAS: Genome-wide association studies; IMF: Intramuscular fat; KEGG: Kyoto Encyclopedia of Genes and Genomes; LD: Longissimus dorsi; PPI: Proteinprotein interaction; PSE: Pale, soft and exudative; QTLs: Quantitative trait loci; RNA-seq: RNA sequencing; STRING: Search Tool for the Retrieval of Interacting Genes; WHC: Water holding capacity

\section{Acknowledgements}

The authors wish to thank Jianghai Pig Breeding Co., Ltd. and the Breeding Swine Quality Supervision and Testing Center, Ministry of Agriculture (Wuhan) for their cooperation in this study.

\section{Authors' contributions}

WJ conceived and designed the experiments. ZX and WC carried out computational analysis and carried out the experimental validations. ZX and WJ wrote the manuscript. WC, WY, LH, WH, and $\mathrm{HH}$ contributed to the sample collecting, sequencing, data analysis, and interpretation of data. All authors read and approved the final manuscript.

\section{Funding}

This study was supported by Shandong Swine Industry Technology System Innovation (SDAIT-08-03), Agricultural Science and Technology Innovation Project of SAAS (CXGC2017B02), and Youth Research Fund of SAAS (2016YQN54). The funding agency provided financial support for the research but was not involved in the design of the study nor collection, analysis, and interpretation of data or in writing the manuscript.

Availability of data and materials

All data generated in this study were included in the main article and its supplementary files. The raw data of RNA sequencing have been deposited in the National Center for Biotechnology Information Sequence Read Archive with accession No. PRJNA527944 (Available online: https://www.ncbi.nlm.nih. gov/sra/PRJNA527944).

Ethics approval and consent to participate

All the animal procedures we used were reviewed and approved by the Institutional Animal Care and Use Committee of Institute of Animal Science and Veterinary Medicine, Shandong Academy of Agricultural Sciences under permit number IACC20060101.

Consent for publication

Not applicable.

Competing interests

The authors declare that they have no competing interests. 
Received: 6 May 2019 Accepted: 22 November 2019

Published online: 02 December 2019

\section{References}

1. Honikel KO. Reference methods for the assessment of physical characteristics of meat. Meat Sci. 1998;49(4):447-57.

2. Seiquer I, Palma-Granados P, Haro A, Lara L, Lachica M, Fernández-Fígares Nieto R. Meat quality traits in longissimus lumborum and gluteus medius muscles from immunocastrated and surgically castrated Iberian pigs. Meat Sci. 2019;150:77-84.

3. Traore S, Aubry L, Gatellier P, Przybylski W, Jaworska D, Kajak-Siemaszko K, Santé-Lhoutellier V. Higher drip loss is associated with protein oxidation. Meat Sci. 2012:90(4):917-24.

4. Kapper C, Walukonis CJ, Scheffler TL, Scheffler JM, Don C, Morgan MT, Forrest JC, Gerrard DE. Moisture absorption early postmortem predicts ultimate drip loss in fresh pork. Meat Sci. 2014;96:971-6.

5. Welzenbach J, Neuhoff C, Heidt H, Cinar MU, Looft C, Schellander K, Tholen E, Große-Brinkhaus C. Integrative analysis of metabolomic, proteomic and genomic data to reveal functional pathways and candidate genes for drip loss in pigs. Int J Mol Sci. 2016;17(9):E1426.

6. Hermesch S, Luxford BG, Graser HU. Genetic parameters for lean meat yield, meat quality, reproduction and feed efficiency traits for Australian pigs 1 Description of traits and heritability estimates. Livest Prod Sci. 2000;65(3): 239-48

7. van Wijk HJ, Arts DJ, Matthews JO, Webster M, Ducro BJ, Knol EF. Genetic parameters for carcass composition and pork quality estimated in a commercial production chain. J Anim Sci. 2005;83(2):324-33.

8. Suzuki K, Irie M, Kadowaki H, Shibata T, Kumagai M, Nishida A. Genetic parameter estimates of meat quality traits in Duroc pigs selected for average daily gain, longissimus muscle area, backfat thickness, and intramuscular fat content. J Anim Sci. 2005;83(9):2058-65.

9. Ma J, Yang J, Zhou L, Zhang Z, Ma H, Xie X, Zhang F, Xiong X, Cui L, Yang $H$. Genome-wide association study of meat quality traits in a white DurocX Erhualian F2 intercross and Chinese Sutai pigs. PLoS One. 2013;8(5):e64047.

10. Casiró S, Velez-Irizarry D, Ernst CW, Raney NE, Bates RO, Charles MG, Steibel JP. Genome-wide association study in an F2 Duroc $\times$ Pietrain resource population for economically important meat quality and carcass traits. $J$ Anim Sci. 2017;95(2):545-58.

11. Schmid M, Maushammer M, Preuß S, Bennewitz J. Mapping QTL for production traits in segregating Piétrain pig populations using genomewide association study results of F2 crosses. Anim Genet. 2018;49(4):317-20.

12. Fujii J, Otsu K, Zorzato F, de Leon S, Khanna VK, Weiler JE, O'Brien PJ, Maclennan $\mathrm{DH}$. Identification of a mutation in porcine ryanodine receptor associated with malignant hyperthermia. Science. 1991;253(5018):448-51.

13. Milan D, Jeon JT, Looft C, Amarger V, Robic A, Thelander M, Rogel-Gaillard C, Paul S, Lundström N, Rask $L$, et al. A mutation in PRKAG3 associated with excess glycogen content in pig skeletal muscle. Science. 2000;288(5469): $1248-51$.

14. Ma J, Yang J, Zhou L, Ren J, Liu X, Zhang H, Yang B, Zhang Z, Ma H, Xie X, et al. A splice mutation in the PHKG1 gene causes high glycogen content and low meat quality in pig skeletal muscle. PLoS Genet. 2014;10(10): e1004710.

15. Xing K, Zhu F, Zhai L, Chen S, Tan Z, Sun Y, Hou Z, Wang C. Identification of genes for controlling swine adipose deposition by integrating transcriptome, whole-genome resequencing, and quantitative trait loci data. Sci Rep. 2016;6:23219.

16. Chen W, Fang G, Wang S, Wang H, Zeng Y. Longissimus lumborum muscle transcriptome analysis of Laiwu and Yorkshire pigs differing in intramuscular fat content. Genes Genom. 2017;39:759-66.

17. Li B, Liu K, Weng Q, Li P, Wei W, Li Q, Chen J, Huang R, Wu W, Liu H. RNAseq analysis reveals new candidate genes for drip loss in a Pietrain $\times$ Duroc x landrace $\times$ Yorkshire population. Anim Genet. 2016:47(2):192-9.

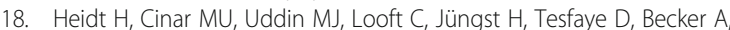
Zimmer A, Ponsuksili S, Wimmers K, et al. A genetical genomics approach reveals new candidates and confirms known candidate genes for drip loss in a porcine resource population. Mamm Genome. 2013;24(9-10):416-26.

19. Voermans NC, Bönnemann CG, Huijing PA, Hamel BC, van Kuppevelt $\mathrm{TH}$, de Haan A, Schalkwijk J, van Engelen BG, Jenniskens GJ. Clinical and molecular overlap between myopathies and inherited connective tissue diseases. Neuromuscul Disord. 2008;18(11):843-56.
20. Lawson $M$. The role of integrin degradation in post-mortem drip loss in pork. Meat Sci. 2004;68(4):559-66.

21. Straadt I, Rasmussen M, Young J, Bertram H. Any link between integrin degradation and water-holding capacity in pork? Meat Sci. 2008:80(3):722-7.

22. Zhang W, Lonergan S, Gardner M, Huff-Lonergan E. Contribution of postmortem changes of integrin, desmin and $\mu$-calpain to variation in water holding capacity of pork. Meat Sci. 2006;74(3):578-85.

23. Midwood K, Orend $G$. The role of tenascin- $C$ in tissue injury and tumorigenesis. J Cell Commun Signal. 2009;3:287-310.

24. van der Flier A, Sonnenberg A. Function and interactions of integrins. Cell Tissue Res. 2001:305(3):285-98.

25. Schips TG, Vanhoutte D, Vo A, Correll RN, Brody MJ, Khalil H, Karch J, Tjondrokoesoemo A, Sargent MA, Maillet M, et al. Thrombospondin-3 augments injury-induced cardiomyopathy by intracellular integrin inhibition and sarcolemmal instability. Nat Commun. 2019;10(1):76.

26. Suleiman M, Abdulrahman N, Yalcin H, Mraiche F. The role of CD44, hyaluronan and NHE1 in cardiac remodeling. Life Sci. 2018;209:197-201.

27. Hwang IH, Park BY, Kim JH, Cho SH, Lee JM. Assessment of postmortem proteolysis by gel-based proteome analysis and its relationship to meat quality traits in pig longissimus. Meat Sci. 2005;69(1):79-91.

28. Di Luca A, Hamill RM, Mullen AM, Slavov N, Elia G. Comparative proteomic profiling of divergent phenotypes for water holding capacity across the post mortem ageing period in porcine muscle exudate. PLoS One. 2016; 11(3):e0150605.

29. Ponsuksili S, Jonas E, Murani E, Phatsara C, Srikanchai T, Walz C, Schwerin M, Schellander K, Wimmers K. Trait correlated expression combined with expression QTL analysis reveals biological pathways and candidate genes affecting water holding capacity of muscle. BMC Genomics. 2008:9:367.

30. Kim D, Langmead B, Salzberg S. HISAT: a fast spliced aligner with low memory requirements. Nat Methods. 2015;12(4):357-60.

31. Liao Y, Smyth G, Shi W. featureCounts: an efficient general purpose program for assigning sequence reads to genomic features. Bioinformatics. 2014; 30(7):923-30.

32. Love Ml, Huber W, Anders S. Moderated estimation of fold change and dispersion for RNA-seq data with DESeq2. Genome Biol. 2014;15(12):550.

33. Yu G, Wang LG, Han Y, He QY. clusterProfiler: an R package for comparing biological themes among gene clusters. OMICS. 2012;16(5):284-7.

\section{Publisher's Note}

Springer Nature remains neutral with regard to jurisdictional claims in published maps and institutional affiliations.

Ready to submit your research? Choose BMC and benefit from:

- fast, convenient online submission

- thorough peer review by experienced researchers in your field

- rapid publication on acceptance

- support for research data, including large and complex data types

- gold Open Access which fosters wider collaboration and increased citations

- maximum visibility for your research: over $100 \mathrm{M}$ website views per year

At BMC, research is always in progress.

Learn more biomedcentral.com/submissions 